\title{
The Differential Role of Human Cationic Trypsinogen (PRSS1) p.R122H Mutation in Hereditary and Nonhereditary Chronic Pancreatitis: A Systematic Review and Meta-Analysis
}

\author{
Cheng Hu, ${ }^{1,2}$ Li Wen, ${ }^{1,3}$ Lihui Deng, ${ }^{1}$ Chenlong Zhang, ${ }^{1}$ Aurelia Lugea, ${ }^{2}$ Hsin-Yuan Su, ${ }^{2}$ \\ Richard T. Waldron, ${ }^{2}$ Stephen J. Pandol, ${ }^{2}$ and Qing Xia ${ }^{1}$ \\ ${ }^{1}$ Department of Integrated Traditional Chinese and Western Medicine, West China Medical School/West China Hospital, \\ Sichuan, China \\ ${ }^{2}$ Division of Gastroenterology, Department of Medicine, Cedars-Sinai Medical Center, Los Angeles, CA, USA \\ ${ }^{3}$ Department of Pediatric Gastroenterology, Children's Hospital of Pittsburgh of UPMC and School of Medicine, \\ University of Pittsburgh, Pittsburgh, PA, USA
}

Correspondence should be addressed to Li Wen; wenli7007@gmail.com, Stephen J. Pandol; stephen.pandol@cshs.org, and Qing Xia; xiaqing@medmail.com.cn

Received 3 May 2017; Revised 22 August 2017; Accepted 7 September 2017; Published 8 October 2017

Academic Editor: Niccola Funel

Copyright (c) 2017 Cheng $\mathrm{Hu}$ et al. This is an open access article distributed under the Creative Commons Attribution License, which permits unrestricted use, distribution, and reproduction in any medium, provided the original work is properly cited.

Background. Environmental factors and genetic mutations have been increasingly recognized as risk factors for chronic pancreatitis (CP). The PRSS1 p.R122H mutation was the first discovered to affect hereditary CP, with $80 \%$ penetrance. We performed here a systematic review and meta-analysis to evaluate the associations of PRSS1 p.R122H mutation with CP of diverse etiology. Methods. The PubMed, EMBASE, and MEDLINE database were reviewed. The pooled odds ratio (OR) with 95\% confidence intervals was used to evaluate the association of p.R122H mutation with CP. Initial analysis was conducted with all etiologies of $\mathrm{CP}$, followed by a subgroup analysis for hereditary and nonhereditary $\mathrm{CP}$, including alcoholic or idiopathic CP. Results. A total of eight case-control studies (1733 cases and 2415 controls) were identified and included. Overall, PRSS1 p.R122H mutation was significantly associated with an increased risk of $\mathrm{CP}(\mathrm{OR}=4.78[1.13-20.20])$. Further analysis showed p.R122H mutation strongly associated with the increased risk of hereditary CP $(\mathrm{OR}=65.52$ [9.09-472.48]) but not with nonhereditary CP, both alcoholic and idiopathic CP. Conclusions. Our study showing the differential role of $\mathrm{p} . \mathrm{R} 122 \mathrm{H}$ mutation in various etiologies of $\mathrm{CP}$ indicates that this complex disorder is likely influenced by multiple genetic factors as well as environmental factors.

\section{Introduction}

Chronic pancreatitis (CP) is a wide range of progressive fibroinflammatory disease of the exocrine pancreas that eventually leads to irreversible impairment of exocrine and endocrine functions of the gland $[1,2]$. The incidence of $\mathrm{CP}$ ranges from 4 to 14 per 100,000 worldwide with a higher incidence reported in recent years [3]. Our understanding of the pathogenesis and pathophysiology of $\mathrm{CP}$, including the development of pancreatic fibrosis, etiologic risk factors, natural history, and genetic and epigenetic changes associated, has significantly advanced over time $[1,2,4,5]$. $\mathrm{CP}$ has traditionally been classified as alcohol, hereditary, obstructive, hyperlipidemia, or idiopathic on the basis of etiology [6]. The most common disease phenotype is described as chronic calcifying pancreatitis, which is characterized by clinically apparent acute pancreatitis (AP) at the early stage, progressive development of intraductal stones, pancreatic ductal distortion, strictures, and pancreatic atrophy and extensive destruction of the pancreatic parenchyma leading to steatorrhea and diabetes [3]. Alcohol and smoking are identified as independent risk factors for CP. Both are 
associated with disease progression, and their risks are likely multiplicative [2]. Several genetic mutations, including human cationic trypsinogen (PRSS) 1, PRSS2, serine protease inhibitor Kazal type 1 gene (SPINK1), chymotrypsinogen C gene (CTRC), transmembrane conductance regulator gene (CFTR), and calcium-sensing receptor (CASR), have been noted as risk factors $[2,6]$. The role of these gene mutations in $\mathrm{CP}$ is becoming increasingly recognized, although the proportion of CP with known genetic mutations is rather small.

PRSS1 mutation was first discovered to be associated with the phenotype of hereditary pancreatitis (HP) twenty years ago [7]. Carriers with this mutation tend to develop recurrent AP with an early onset of the disease (prior to the second decade of life) and the development of chronic pancreatitis and also have a significantly increased risk for development of pancreatic adenocarcinoma $[8,9]$. $\mathrm{R} 122 \mathrm{H}$ mutation of the PRSS1 gene, detected in more than $50 \%$ of the patients with HP, was the most common mutation in patients with $\mathrm{HP}$ [10]. A single $\mathrm{G}$ to A transition mutation in the cationic trypsinogen gene in exon 3 resulted in an arginine (CGC) to histidine (CAC) substitution at amino acid residue 117 of trypsinogen (p.R122H) and this amino acid change eliminates a trypsin cleavage site on the surface of trypsinogen and is predicted to prevent trypsinogen autoinactivation $[7,11]$. Mice expressing mouse PRSS1 mutant p.R122H transgene exhibited early onset of acinar cell injury and inflammation that progressed with age to a chronic stage and associated with fibrosis and acinar cell differentiation [11]. Expression of human PRSS1 mutants (p.R122H or p.N29I) in murine pancreatic acinar cells also promoted pancreatic injury [12]. Interestingly, endogenously activated trypsinogen (PACE-trypsinogen) in acinar cells caused spontaneous development of AP, but did not exhibit the features of CP, including fibrosis [13]. Genetic deletion of trypsinogen 7 , a mouse cationic trypsinogen, caused a $50 \%$ reduction in pancreatic damage in caerulein-induced AP [14], but exhibited the same degree of CP induced by repetitive challenges of caerulein compared to wild-type mice [15].

In this study, we performed a systematic review and metaanalysis to investigate the association between p.R122H mutation in PRSS1 gene and the risk of CP. We used PRSS1 p.R122H as an example of genetic risk factor in order to understand more about the association of single genetic factor in CP patients. We found that PRSS1 p.R122H mutation was significantly associated with an increased risk of hereditary CP. Further analysis suggested p.R122H mutation was weakly but still significantly associated with an increased risk of nonhereditary $\mathrm{CP}$, including alcoholic or idiopathic CP with a slightly greater risk in idiopathic CP.

\section{Methods}

2.1. Search Strategy. A search of the literature was conducted by two authors (C.H and L.W) independently using PubMed, EMBASE, and MEDLINE electronic databases. The keywords PRSS1 or p.R122H were combined with pancreatitis. The filter "human" was applied. The search was conducted using database entries from January 1996 to July 2016. The language was limited to English. The duplicates were removed manually. We also reviewed reference list of citations in the identified publications for additional citations.

2.2. Eligibility Criteria. The published observational studies found by our search that reported the association of chronic pancreatitis with p. $\mathrm{R} 122 \mathrm{H}$ mutation were included in this review. Studies that enrolled pediatric patients were excluded $(n=36)$. All basic and animal experimental studies were also excluded $(n=26)$. Review articles $(n=67)$ and case reports $(n=39)$ were excluded. Studies that investigated autoimmune pancreatitis $(n=1)$ or recurrent acute pancreatitis $(n=3)$ were also excluded.

2.3. Data Extraction. Data were extracted by two authors (C. H and L. W) independently for each individual study. The following data were extracted: year, study design, racial background, genotyping method, criteria used for diagnosis of $\mathrm{CP}$, number of patients diagnosed as $\mathrm{CP}$, total number of patients, etiology of CP (alcohol, idiopathic, hereditary, and other), history of alcohol consumption, and history of smoking if applicable.

2.4. Quality Assessment. The quality of included case-control studies was systematically assessed using the Newcastle Ottawa Scale (NOS). A maximum of 9 points were given to each study within the categories of the selection, comparability, and exposure, as scored by two independent observers. Studies were considered to be "good" quality if they scored $>5$ points, whereas studies were considered to be "poor" quality if they received $\leq 5$ points.

2.5. Definitions. The American Pancreatic Association (APA) guidelines [2] were used to define disease categories. Hereditary CP was defined as present in an individual with CP and with one or two affected first-degree relatives or two or more second-degree relatives affected [16-19]. Nonhereditary CP included idiopathic CP, alcoholic CP, and other etiologies. Idiopathic CP was exclusively diagnosed in the absence of known etiological factors such as alcohol, gallstone, infection, trauma, medications, age over 65 , and a positive family history [6].

Alcohol was considered the cause of $\mathrm{CP}$ in those who consumed more than 60 or $80 \mathrm{~g}$ per day for at least 2 years. Smokers were defined as those who smoked 10 or more cigarettes per day for at least 2 years.

2.6. Data Analysis. For each study, the association between PRSS1 p.R122H mutation and the risk of CP was initially evaluated. A preliminary meta-analysis combining all studies included regardless of etiology was conducted. Further stratified analysis was used to assess the risk of hereditary and nonhereditary CP with PRSS1 p.R122H mutation, including subanalysis of alcoholic and idiopathic CP. Forest plots were created showing the pooled odds ratio (OR) with the corresponding 95\% confidence intervals (CIs). Data were pooled using the random effects model to provide a more conservative estimate. Statistical heterogeneity between studies was determined with Cochran $Q$ test and the $I^{2}$ value [20]. High statistical heterogeneity was defined as $>70 \%$, medium heterogeneity was defined as $50 \%-70 \%$, and low heterogeneity 

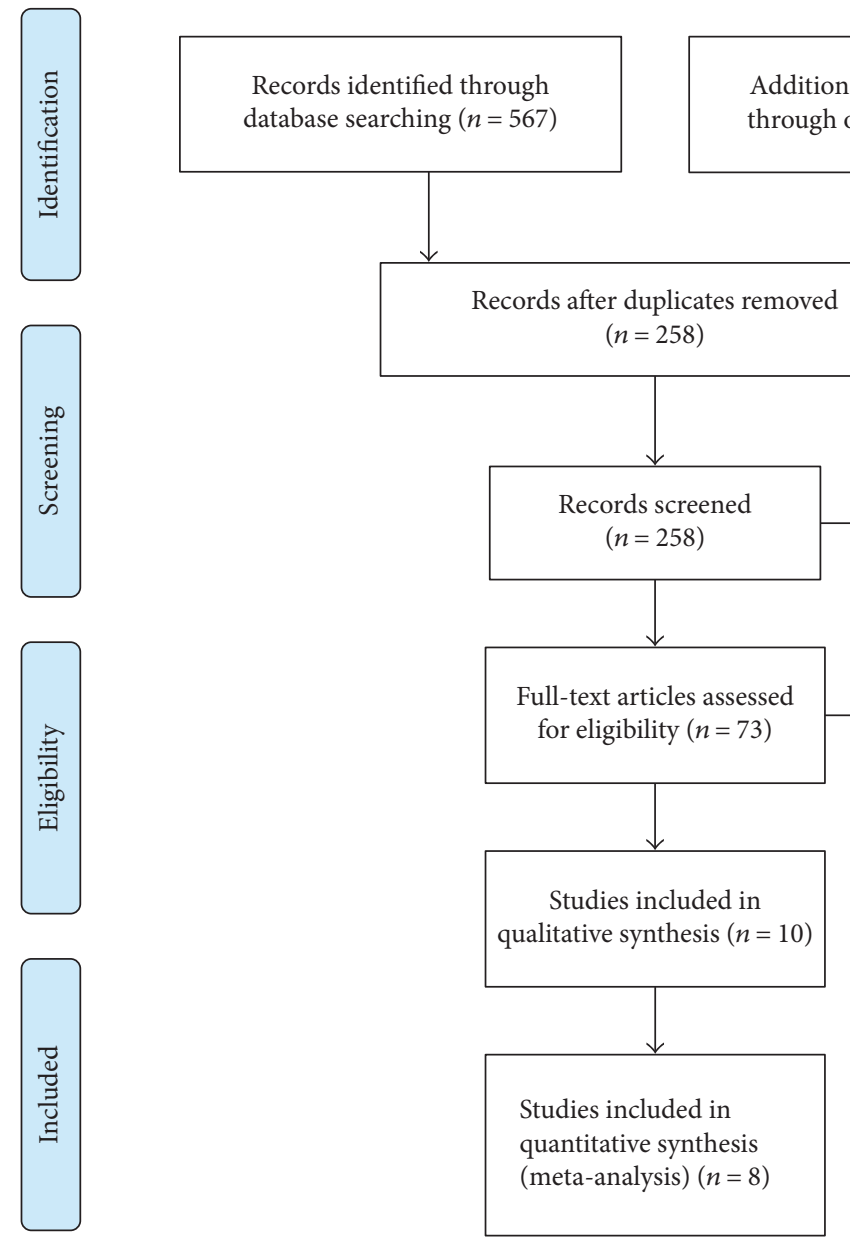

database searching $(n=567)$

Additional records identified

through other sources $(n=0)$

FIgURE 1: Flow chart of study selection [37].

was defined as $0 \%-50 \%$ [20]. A $p$ value of $\leq 0.05$ was considered as statistical significance. Sensitivity analysis was used to test the robustness of associations by omitting each individual study in turn from all available studies when appropriate. Publication bias was assessed by visual inspection of funnel plot [21]. The analysis was performed by Stata software, version SE/13.0 (Stata Corp LP, College Station, Texas, USA).

\section{Results}

3.1. Study Selection. A total of 567 studies were retrieved from PubMed, EMBASE, and MEDLINE. After removal of duplicates, 258 studies were screened by the title and abstracts. In the preliminary screening, we excluded 185 studies because of the following reasons: pediatric patients $(n=36)$, animal and experimental studies $(n=28)$, case reports $(n=39)$, cross-sectional studies $(n=1)$, review $(n=67)$, editorial $(n=2)$, and others $(n=12)$. After removing, seventy-three articles potentially met the inclusion criteria for eligibility, and 63 were excluded for the reasons as follows: not relevant $(n=46)$, no genotyping for PRSS1 $(n=7)$, no genotyping for p.R122H $(n=6)$, recurrent $\mathrm{AP}(n=3)$, and autoimmune pancreatitis $(n=1)$. Overall, ten studies [16-19, 22-27] were identified and scored for assessing the quality. Two studies were excluded from meta-analysis due to quality score $\leq 5$. Finally, eight studies fulfilled completely the criteria for inclusion in this current meta-analysis. The flow diagram showing the study selection process is presented in Figure 1. Methodological quality assessment of the included studies is shown in Supplementary Table 1 available online at https://doi.org/10.1155/2017/9505460.

3.2. Study Characteristics. A total of eight case-controldesigned studies including CP patients were selected. Table 1 shows information published for these studies: ethnicity, genotyping methods, and etiologies of CP. Six studies were conducted in Europe and the other two in Asia. First, we analyzed the eight studies that included a total of 1733 cases and 2415 controls. Then, we analyzed the cases based on whether subjects had HP or not (non-HP). Finally, four studies including HP patients (255 cases and 2214 controls) and all eight studies including non-HP patients (1478 cases and 2415 controls) were enrolled. Further analysis of non-HP patients was carried out using six of the studies with ACP patients (308 cases and 575 controls) and seven of the studies with ICP patients (1170 cases and 2315 controls). 
TABLE 1: Characteristics of case-control studies included.

\begin{tabular}{|c|c|c|c|c|c|c|c|}
\hline \multirow{2}{*}{ Author, year } & \multirow{2}{*}{ Journal } & \multirow{2}{*}{ Genotyping method } & \multirow{2}{*}{ Author country } & \multirow{2}{*}{ Study population } & \multicolumn{2}{|c|}{ Non-HP } & \multirow{2}{*}{$\mathrm{HP}$} \\
\hline & & & & & ACP & ICP & \\
\hline Sisman et al., 2015 [27] & Turk J Gastroenterol & RFLP-PCR & Turkey & Turkey & + & + & \\
\hline Madro et al., 2015 [26] & Gastroenterol Res Pract & RT-PCR & Poland & N/A & + & & \\
\hline Rosendahl et al., 2013 [16] & Gut & PCR & Germany & German & & + & + \\
\hline Gasiorowska et al., 2011 [25] & Dig Dis Sci & RFLP-PCR & Poland & Polish & + & + & \\
\hline Mora et al., 2009 [17] & Pancreatology & RFLP-PCR & Spain & Spanish & + & + & + \\
\hline Chandak 2004 [18] & Gut & - & India & Indian & + & + & + \\
\hline Teich et al., 2002 [19] & Am J Gastroenterol & PCR & Germany & N/A & & + & + \\
\hline O’Reilly et al., 2001 [22] & Digestion & PCR & UK & Ethnically matched & + & + & \\
\hline
\end{tabular}

N/A: not available.

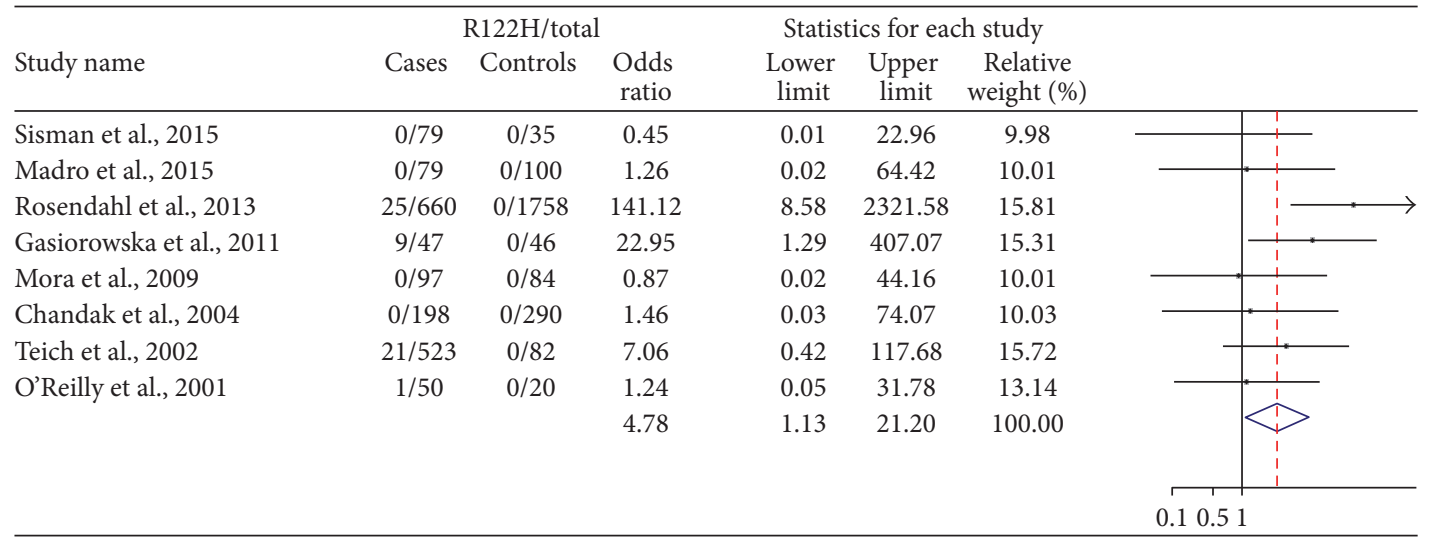

FIGURE 2: Forest plots showing the association between chronic pancreatitis with all etiologies combined and R122H mutation incidence.

3.3. Chronic Pancreatitis with All Etiologies Combined. Initial meta-analysis of individual studies and the combined results of the eight studies with all etiologies of CP are shown in Figure 2. The total number of patients from these studies was $1733 \mathrm{CP}$ cases with 2415 healthy controls. The weighted OR revealed an association between p.R122H mutation and that the risk of $\mathrm{CP}$ is $4.78(1.13-20.20)$. There was low heterogeneity detected between the studies $(p=0.171$ and $\left.I^{2}=32.2 \%\right)$.

3.4. Hereditary Chronic Pancreatitis versus Nonhereditary Chronic Pancreatitis. Four of the studies assessed patients with hereditary CP (255 cases and 2214 controls, Figure 3(a)). The p.R122H mutation was detected in 39 of 255 cases and none in 2214 controls. There was low heterogeneity detected between the studies $\left(p=0.235\right.$ and $I^{2}=$ $29.5 \%)$. The pooled OR revealed an association between p.R122H mutation and the risk of hereditary CP of 65.52 (9.09-472.48). Eight studies evaluated patients with nonhereditary CP (1478 cases and 2415 controls, Figure 3(b)). The p.R122H mutation was present in 8 of 1478 cases and none in 2415 controls. There was low heterogeneity detected between the studies $\left(p=0.241\right.$ and $\left.I^{2}=23.6 \%\right)$. The pooled OR revealed an association between p.R122H mutation, and an increased risk of nonhereditary CP is 2.79 (95\%CI, $0.68-11.55)$. These data indicate that $\mathrm{p} . \mathrm{R} 122 \mathrm{H}$ mutation is strongly associated with hereditary CP and has a weak association with nonhereditary $\mathrm{CP}$.

3.5. Subgroup Analysis of Alcoholic and Idiopathic CP. Six studies assessed patients with alcoholic CP (308 cases and 575 controls, Figure 4). The p.R122H mutation was detected in 8 of 308 cases and none in 575 controls. There was no heterogeneity detected between the studies $(p=0.662$ and $\left.I^{2}=0.0 \%\right)$. The pooled OR of $3.39(0.79-14.54)$ revealed a trend that p.R122H mutation may be associated with an increased risk of alcoholic CP. Since there is only one study with a small size suggesting an association between p.R122H mutation and alcoholic CP and 95\%CI crossed 1 , we think the p.R122H mutation may not be associated with alcoholic CP, although further study is required to confirm these findings.

Seven studies assessed patients with idiopathic CP (1170 cases and 2315 controls, Figure 5). The p.R122H mutation was detected in 9 of 1170 cases and none in 2315 controls. There was low heterogeneity detected between the studies $\left(p=0.330\right.$ and $\left.I^{2}=13.1 \%\right)$. The pooled OR is $4.43(1.03-$ 19.05), which revealed a significant weak association between p.R122H mutation and an increased risk of idiopathic CP. Consistent with the analysis in nonhereditary $\mathrm{CP}$, there was a weak but still significant association between p.R122H mutation and an increased risk of CP. 


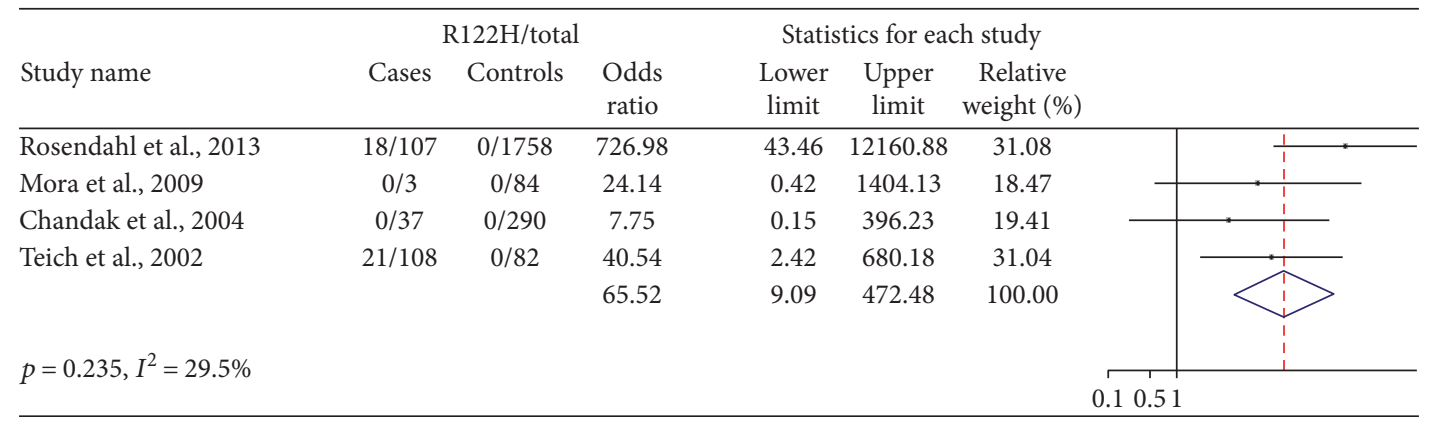

(a)

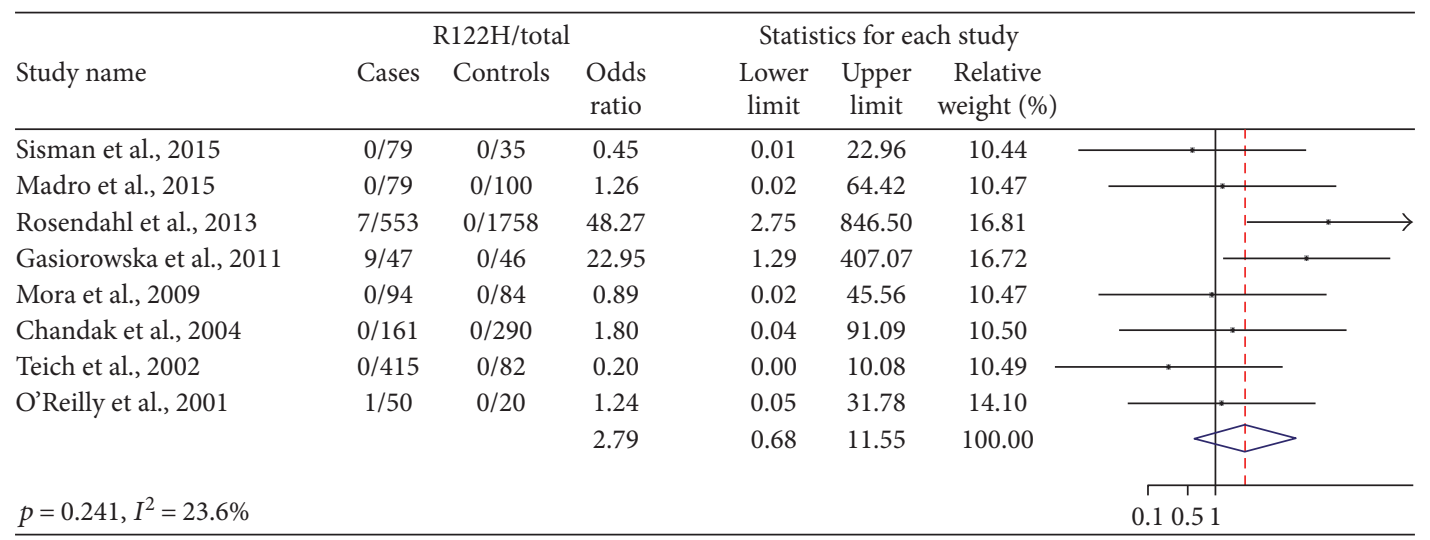

(b)

FIGURE 3: Hereditary CP/R122H mutation versus nonhereditary CP/R122H mutation for case-control studies.

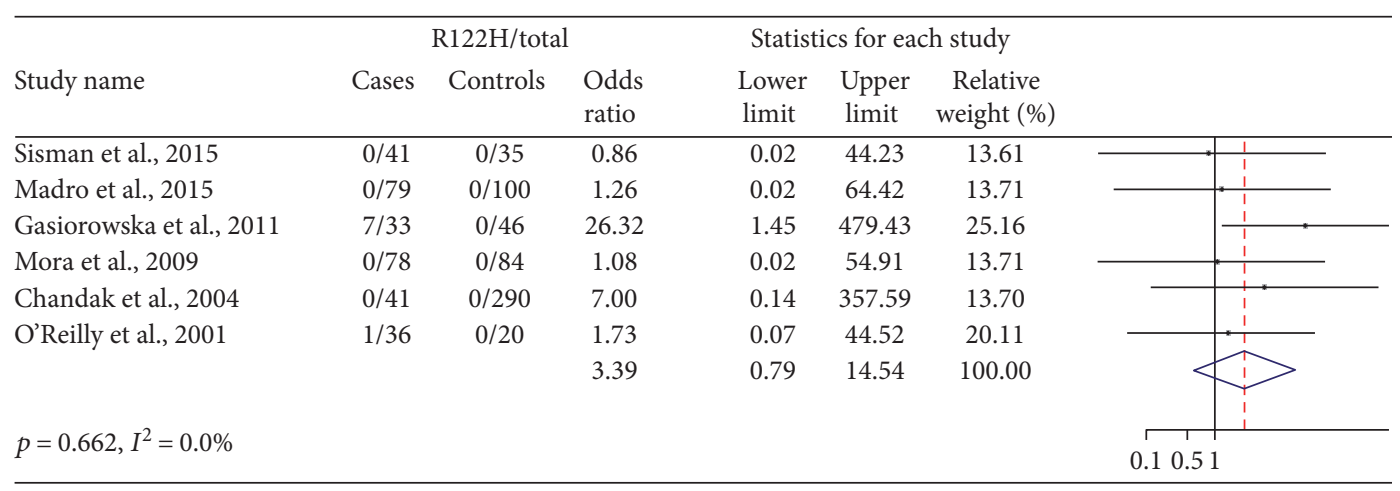

FIGURE 4: Alcoholic CP/R122H mutation for case-control studies.

The sensitivity analysis was performed by sequential omission of each single study in turn. The results indicated that removal of any individual studies one at a time did not change the direction of the pooled effect size (Supplementary Figure 1), indicating that our results are relatively stable and credible.

\section{Discussion}

Overall, we found that the PRSS1 p.R122H mutation is significantly associated with an increased risk of CP. Our finding is consistent with the meta-analysis conducted by Liu and
Zhang [28] which suggested a significant association between total pancreatitis and the PRSS1 gene. But this study neither specified the sites of the gene mutations nor compared the different pancreatitis etiologies. In our current study, we specifically asked whether there is an association between PRSS1 p. R122H and chronic pancreatitis with subgroup analysis applied to the known etiologies. Further analysis showed that p.R122H mutation is strongly associated with hereditary CP. Since the first report in 1996 indicating that PRSS1 p.R122H mutation was associated with the phenotype of HP in a North American family [7], strong associations between the p.R122H mutation in the PRSS1 gene and HP 


\begin{tabular}{|c|c|c|c|c|c|c|c|}
\hline \multirow[b]{2}{*}{ Study name } & \multicolumn{3}{|c|}{$\mathrm{R} 122 \mathrm{H} /$ total } & \multicolumn{3}{|c|}{ Statistics for each study } & \\
\hline & Cases & Controls & $\begin{array}{l}\text { Odds } \\
\text { ratio }\end{array}$ & $\begin{array}{l}\text { Lower } \\
\text { limit }\end{array}$ & $\begin{array}{l}\text { Upper } \\
\text { limit }\end{array}$ & $\begin{array}{c}\text { Relative } \\
\text { weight (\%) }\end{array}$ & \\
\hline Sisman et al., 2015 & $0 / 38$ & $0 / 35$ & 0.92 & 0.02 & 47.72 & 12.13 & \\
\hline Rosendahl et al., 2013 & $7 / 553$ & $0 / 1758$ & 48.27 & 2.75 & 846.50 & 20.93 & \\
\hline Gasiorowska et al., 2011 & $2 / 14$ & $0 / 46$ & 18.60 & 0.84 & 412.87 & 18.39 & \\
\hline Mora et al., 2009 & $0 / 16$ & $0 / 84$ & 5.12 & 0.10 & 267.40 & 12.09 & \\
\hline Chandak et al., 2004 & $0 / 120$ & $0 / 290$ & 2.41 & 0.05 & 122.21 & 12.25 & \\
\hline Teich et al., 2002 & $0 / 415$ & $0 / 82$ & 0.20 & 0.00 & 10.08 & 12.23 & \\
\hline \multirow[t]{2}{*}{ O’Reilly et al., 2001} & $0 / 14$ & $0 / 20$ & 1.41 & 0.03 & 75.46 & 11.97 & \\
\hline & & & 4.43 & 1.03 & 19.05 & 100.00 & \\
\hline$p=0.330, I^{2}=13.1 \%$ & & & & & & & 0.10 .51 \\
\hline
\end{tabular}

Figure 5: Idiopathic CP/R122H mutation for case-control studies.

have been reported in a number of studies from many parts of the world including Europe and Asia [29-31]. Subgroup analysis for the known etiologies, including alcoholic and idiopathic CP, showed that p. $\mathrm{R} 122 \mathrm{H}$ mutation is weakly correlated with an increased risk of nonhereditary CP. In recent years, smoking has been recognized as an independent risk factor affecting the course and progression of $\mathrm{CP}$ $[32,33]$. We were unable to investigate the association of smoking with the risk of $\mathrm{CP}$ as there is lack of reported data extracted from the studies we included in our analysis.

Various PRSS1 gene mutations in exon 2 and exon 3 have been reported to be pathogenic and associated with the phenotype of CP. p.R122H and p.N29I mutations are the most common mutations [34]. Recently, the genome-wide association study (GWAS) in alcoholic CP discovered that the common genetic variants in the PRSS1-PRSS2 locus account for an increased risk for alcoholic and sporadic CP, but rare PRSS1 gene variants, including three known diseases that were also screened and identified, were not associated with the observed phenotype [35]. This finding was replicated and refined in a large European cohort by an independent group [36]. Both studies suggested the importance of continuing to identify disease-associated genetic variants.

In summary, our findings indicate that the PRSS1 p.R122H mutation is significantly associated with an increased risk of CP overall. The differential role of PRSS1 p.R122H mutation was observed in both hereditary and nonhereditary CP. These data suggest that multiple genetic and environmental factors determine the initiation and progression of CP.

\section{Disclosure}

This work was presented at the 2017 Digestive Disease Week (DDW) held in Chicago, Illinois. The funders had no role in the study design, data collection and analysis, decision to publish, or preparation of the manuscript.

\section{Conflicts of Interest}

The authors declare no conflicts of interest.

\section{Authors' Contributions}

Cheng Hu, Li Wen, Stephen J. Pandol, and Qing Xia designed the study. Cheng $\mathrm{Hu}$ and $\mathrm{Li}$ Wen performed the work and analyzed the data. Lihui Deng, Chenlong Zhang, Aurelia Lugea, Hsin-Yuan Su, and Richard T. Waldron made technical and scientific contributions. Cheng $\mathrm{Hu}, \mathrm{Li}$ Wen, Stephen J. Pandol, and Qing Xia wrote the paper. All the authors approved the final version. Cheng $\mathrm{Hu}$ and $\mathrm{Li}$ Wen are co-first authors.

\section{Acknowledgments}

This study was supported by the Major Program from Science and Technology Department of Sichuan Province (no. 2015SZ0229/0040205301D51), the Doctoral Program of Higher Education of China (no. 20130181120050), and the China Scholarship Council (no. 201506240063).

\section{References}

[1] H. Witt, M. V. Apte, V. Keim, and J. S. Wilson, "Chronic pancreatitis: challenges and advances in pathogenesis, genetics, diagnosis, and therapy," Gastroenterology, vol. 132, no. 4, pp. 1557-1573, 2007.

[2] D. L. Conwell, L. S. Lee, D. Yadav et al., “American pancreatic association practice guidelines in chronic pancreatitis: evidence-based report on diagnostic guidelines," Pancreas, vol. 43, no. 8, pp. 1143-1162, 2014.

[3] S. Majumder and S. T. Chari, "Chronic pancreatitis," Lancet, vol. 387, no. 10031, pp. 1957-1966, 2016.

[4] E. Aoun, C. C. Chang, J. B. Greer, G. I. Papachristou, M. M. Barmada, and D. C. Whitcomb, "Pathways to injury in chronic pancreatitis: decoding the role of the high-risk SPINK1 N34S haplotype using meta-analysis," PLoS One, vol. 3, no. 4, article e2003, 2008.

[5] D. Yadav and A. B. Lowenfels, "The epidemiology of pancreatitis and pancreatic cancer," Gastroenterology, vol. 144, no. 6, pp. 1252-1261, 2013.

[6] B. Etemad and D. C. Whitcomb, "Chronic pancreatitis: diagnosis, classification, and new genetic developments," Gastroenterology, vol. 120, no. 3, pp. 682-707, 2001. 
[7] D. C. Whitcomb, M. C. Gorry, R. A. Preston et al., "Hereditary pancreatitis is caused by a mutation in the cationic trypsinogen gene," Nature Genetics, vol. 14, no. 2, pp. 141-145, 1996.

[8] K. L. Raphael and F. F. Willingham, "Hereditary pancreatitis: current perspectives," Clinical and Experimental Gastroenterology, vol. 9, pp. 197-207, 2016.

[9] F. U. Weiss, "Pancreatic cancer risk in hereditary pancreatitis," Frontiers in Physiology, vol. 5, p. 70, 2014.

[10] N. Howes, M. M. Lerch, W. Greenhalf et al., "Clinical and genetic characteristics of hereditary pancreatitis in Europe," Clinical Gastroenterology and Hepatology, vol. 2, no. 3, pp. 252-261, 2004.

[11] H. Archer, N. Jura, J. Keller, M. Jacobson, and D. Bar-Sagi, “A mouse model of hereditary pancreatitis generated by transgenic expression of R122H trypsinogen," Gastroenterology, vol. 131, no. 6, pp. 1844-1855, 2006.

[12] T. Athwal, W. Huang, R. Mukherjee et al., "Expression of human cationic trypsinogen (PRSS1) in murine acinar cells promotes pancreatitis and apoptotic cell death," Cell Death and Disease, vol. 5, article e1165, 2014.

[13] B. Ji, S. Gaiser, X. Chen, S. A. Ernst, and C. D. Logsdon, "Intracellular trypsin induces pancreatic acinar cell death but not NF- $\kappa \mathrm{B}$ activation," The Journal of Biological Chemistry, vol. 284, no. 26, pp. 17488-17498, 2009.

[14] R. Dawra, R. P. Sah, V. Dudeja et al., "Intra-acinar trypsinogen activation mediates early stages of pancreatic injury but not inflammation in mice with acute pancreatitis," Gastroenterology, vol. 141, no. 6, pp. 2210-2217.e2, 2011.

[15] R. P. Sah, V. Dudeja, R. K. Dawra, and A. K. Saluja, "Ceruleininduced chronic pancreatitis does not require intra-acinar activation of trypsinogen in mice," Gastroenterology, vol. 144, no. 5, pp. 1076-1085.e2, 2013.

[16] J. Rosendahl, O. Landt, J. Bernadova et al., "CFTR, SPINK1, CTRC and PRSS1 variants in chronic pancreatitis: is the role of mutated CFTR overestimated?," Gut, vol. 62, no. 4, pp. 582-592, 2013.

[17] J. Mora, L. Comas, E. Ripoll et al., "Genetic mutations in a Spanish population with chronic pancreatitis," Pancreatology, vol. 9, no. 5, pp. 644-651, 2009.

[18] G. R. Chandak, "Absence of PRSS1 mutations and association of SPINK1 trypsin inhibitor mutations in hereditary and non-hereditary chronic pancreatitis," Gut, vol. 53, no. 5, pp. 723-728, 2004.

[19] N. Teich, N. Bauer, J. Mossner, and V. Keim, "Mutational screening of patients with nonalcoholic chronic pancreatitis: identification of further trypsinogen variants," The American Journal of Gastroenterology, vol. 97, no. 2, pp. 341-346, 2002.

[20] J. P. Higgins, S. G. Thompson, J. J. Deeks, and D. G. Altman, "Measuring inconsistency in meta-analyses," British Medical Journal, vol. 327, no. 7414, pp. 557-560, 2003.

[21] M. Egger, G. Davey Smith, M. Schneider, and C. Minder, "Bias in meta-analysis detected by a simple, graphical test," British Medical Journal, vol. 315, no. 7109, pp. 629-634, 1997.

[22] D. A. O’Reilly, B. M. Yang, J. E. Creighton, A. G. Demaine, and A. N. Kingsnorth, "Mutations of the cationic trypsinogen gene in hereditary and non-hereditary pancreatitis," Digestion, vol. 64, no. 1, pp. 54-60, 2001.

[23] S. Keiles and A. Kammesheidt, "Identification of CFTR, PRSS1, and SPINK1 mutations in 381 patients with pancreatitis," Pancreas, vol. 33, no. 3, pp. 221-227, 2006.
[24] Q. C. Liu, F. Gao, Q. S. Ou et al., "Novel mutation and polymorphism of PRSS1 gene in the Chinese patients with hereditary pancreatitis and chronic pancreatitis," Chinese Medical Journal, vol. 121, no. 2, pp. 108-111, 2008.

[25] A. Gasiorowska, R. Talar-Wojnarowska, L. Czupryniak et al., "The prevalence of cationic trypsinogen (PRSS1) and serine protease inhibitor, Kazal type 1 (SPINK1) gene mutations in Polish patients with alcoholic and idiopathic chronic pancreatitis," Digestive Diseases and Sciences, vol. 56, no. 3, pp. 894901, 2011.

[26] A. Madro, M. Ciesielka, K. Celinski et al., "The genetic predisposition and its impact on the diabetes mellitus development in patients with alcoholic chronic pancreatitis," Gastroenterology Research and Practice, vol. 2015, Article ID 309156, 5 pages, 2015.

[27] G. Sisman, M. Tugcu, K. Ayla, O. Sebati, and H. Senturk, "Mutation analysis of PRSS1, SPINK1 and CFTR gene in patients with alcoholic and idiopathic chronic pancreatitis: a single center study," The Turkish Journal of Gastroenterology, vol. 26, no. 2, pp. 176-180, 2015.

[28] J. Liu and H. X. Zhang, "A comprehensive study indicates PRSS1 gene is significantly associated with pancreatitis," International Journal of Medical Sciences, vol. 10, no. 8, pp. 981987, 2013.

[29] M. Otsuki, I. Nishimori, T. Hayakawa et al., "Hereditary pancreatitis: clinical characteristics and diagnostic criteria in Japan," Pancreas, vol. 28, no. 2, pp. 200-206, 2004.

[30] V. Rebours, M. C. Boutron-Ruault, M. Schnee et al., "The natural history of hereditary pancreatitis: a national series," Gut, vol. 58, pp. 97-103, 2009.

[31] X. T. Sun, L. H. Hu, T. Xia et al., "Clinical features and endoscopic treatment of Chinese patients with hereditary pancreatitis," Pancreas, vol. 44, no. 1, pp. 59-63, 2015.

[32] S. J. Sankaran, A. Y. Xiao, L. M. Wu, J. A. Windsor, C. E. Forsmark, and M. S. Petrov, "Frequency of progression from acute to chronic pancreatitis and risk factors: a meta-analysis," Gastroenterology, vol. 149, no. 6, pp. 1490-1500.e1, 2015.

[33] J. B. Greer, E. Thrower, and D. Yadav, "Epidemiologic and mechanistic associations between smoking and pancreatitis," Current Treatment Options in Gastroenterology, vol. 13, no. 3, pp. 332-346, 2015.

[34] B. C. Nemeth and M. Sahin-Toth, "Human cationic trypsinogen (PRSS1) variants and chronic pancreatitis," American Journal of Physiology. Gastrointestinal and Liver Physiology, vol. 306, no. 6, pp. G466-G473, 2014.

[35] D. C. Whitcomb, J. LaRusch, A. M. Krasinskas et al., "Common genetic variants in the CLDN2 and PRSS1-PRSS2 loci alter risk for alcohol-related and sporadic pancreatitis," Nature Genetics, vol. 44, no. 12, pp. 1349-1354, 2012.

[36] M. H. Derikx, P. Kovacs, M. Scholz et al., "Polymorphisms at PRSS1-PRSS2 and CLDN2-MORC4 loci associate with alcoholic and non-alcoholic chronic pancreatitis in a European replication study," Gut, vol. 64, pp. 1426-1433, 2015.

[37] D. Moher, A. Liberati, J. Tetzlaff, D. G. Altman, and The PRISMA Group, "Preferred Reporting Items for Systematic Reviews and Meta-Analyses: The PRISMA Statement," PLoS Medicine, vol. 6, no. 7, article e1000097, 2009. 


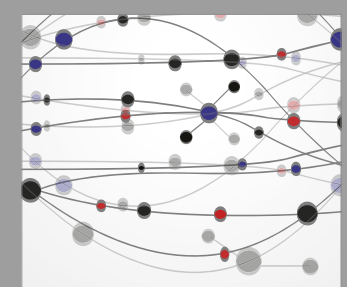

The Scientific World Journal
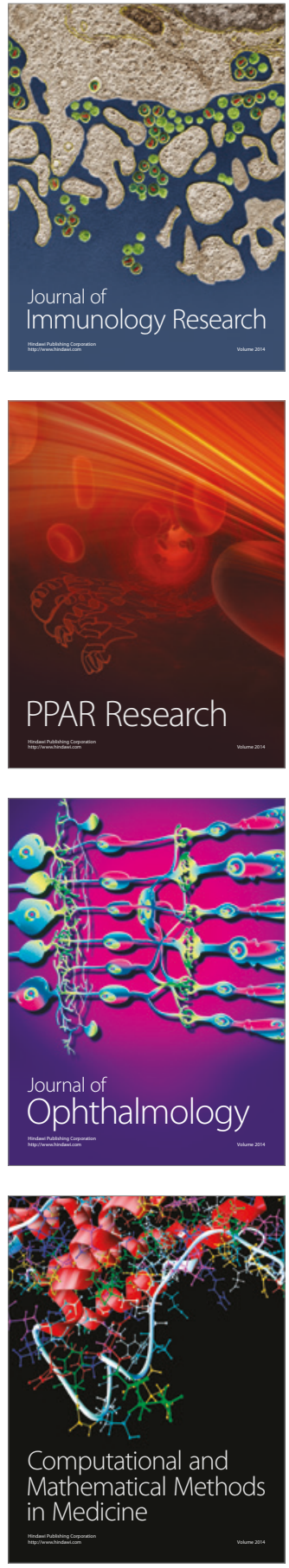

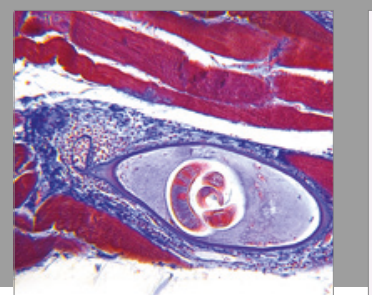

Gastroenterology Research and Practice
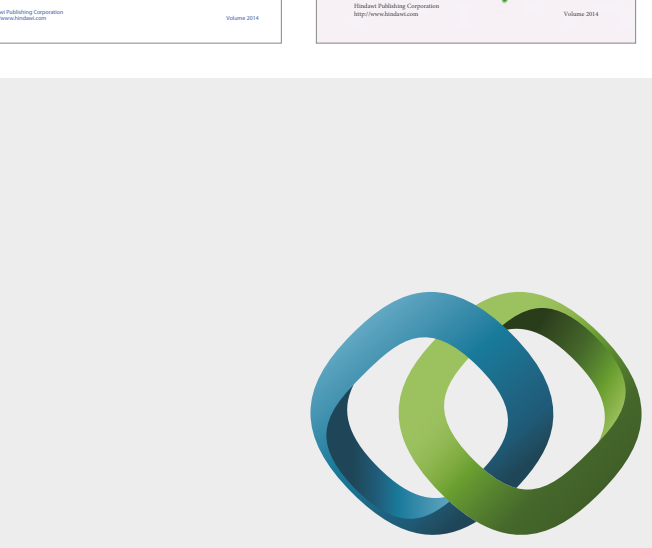

\section{Hindawi}

Submit your manuscripts at

https://www.hindawi.com
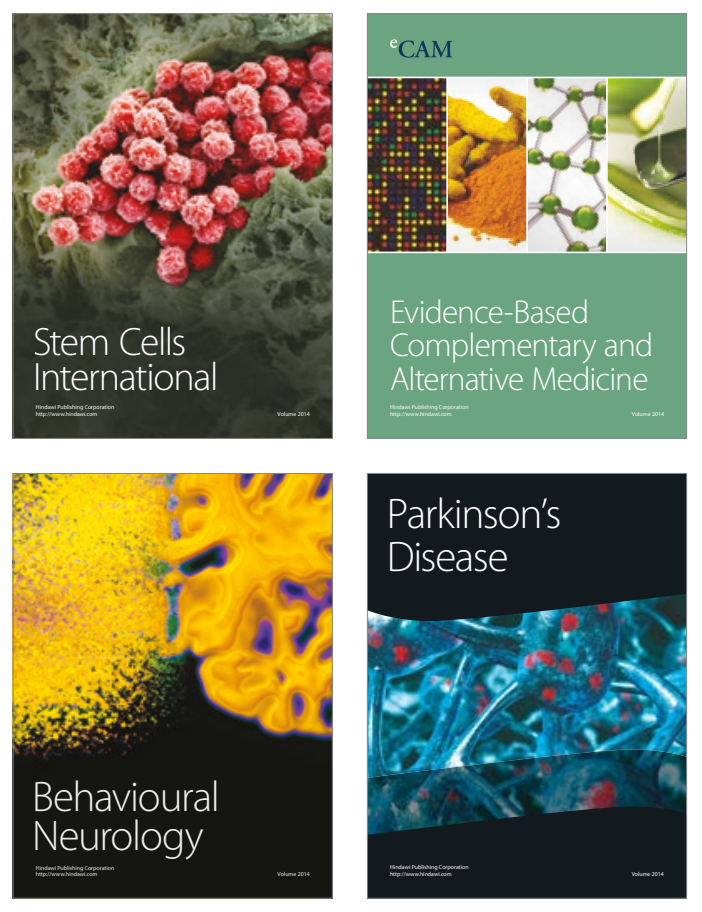
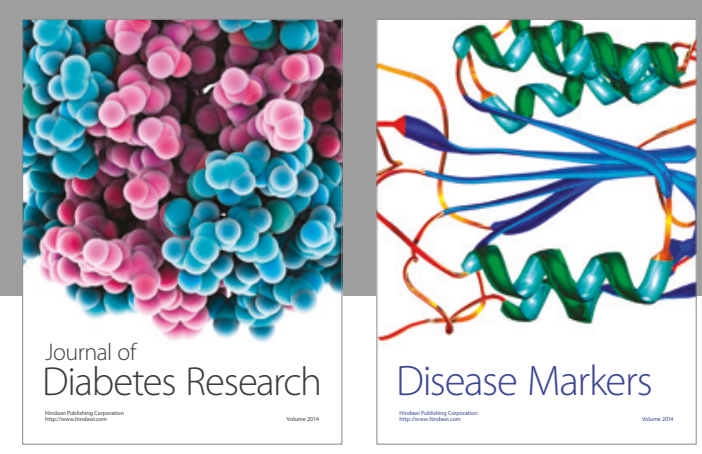

Disease Markers
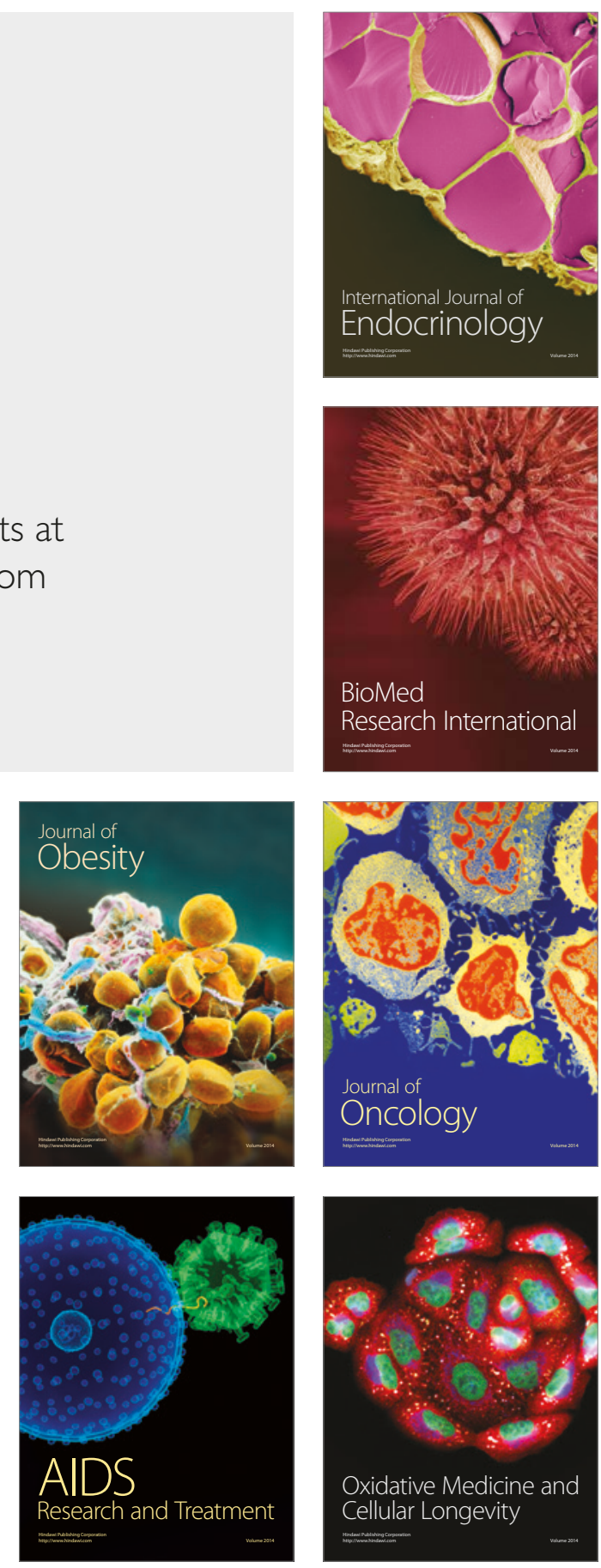